\title{
Experimental Investigation of the Dynamic Effects of Time-Varying Plasma on Low- Frequency Electromagnetic Wave Propagation
}

\author{
Bin Sun, Kai Xie, Yan Liu, Yujin Zhang, Shaoshuai Guo, Ping Ma
}

\begin{abstract}
In this paper, we reveal the dynamic effects of timevarying plasma on low-frequency (LF) electromagnetic (EM) wave propagation. The time-varying attenuation, time-varying phase shift, and power spectrum of the LF EM waves are presented. By using the frequency division multiplexing method, LF EM waves at the four discrete frequencies simultaneously penetrate through a shared time-varying plasma in one shock tube experiment. The experimental results indicate that the time-varying attenuation and phase shift of the LF EM wave are determined by the timevarying behavior of the plasma, and they are positively related to the electron density and frequency of the LF EM waves. The theoretical results are well consistent with the experimental results. Moreover, in the frequency domain, the time-varying plasma causes spectrum expansion of the LF EM waves. These results help us deeply understand the propagation process of LF EM waves in time-varying plasma and design LF communication systems.

Index Terms - EM propagation in plasma media, Time-varying plasma channel, Dynamic effects, Shock tube, Plasma sheath.
\end{abstract}

\section{INTRODUCTION}

$\mathrm{W}$ hen a hypersonic vehicle reenters the atmosphere, air around the vehicle is dissociated and ionized by strong aerodynamic heating. The ionized gas, called the plasma sheath, attenuates and reflects the electromagnetic (EM) waves used for telecommunication, navigation, guidance, and control. As a result, the communications between the vehicle and ground stations are cut off, which is often known as "radio blackout" [1]-[4]. Several methods have been proposed to mitigate radio blackout, such as electrophilic injection [5], magnetic field control [6], the high-frequency (HF) propagation method [7], and the low-frequency (LF) propagation method [8].

In the last decade, the LF propagation method has been considered a potentially feasible method for maintaining lowrate communications [8]-[11]. The EM wave frequency in the LF propagation method (in the range of $0.1 \mathrm{MHz}$ to $30 \mathrm{MHz}$ ) is much lower than the plasma frequency, for which the wavelength is much larger than the size of the plasma sheath. To deeply understand the physical process of the interactions between LF EM waves and the plasma sheath, several propagation models were proposed, including flat plasma [8], spherical shell plasma [9], and cylindrical enveloping plasma [10]. At the same time, corresponding numerical simulations and experiments were conducted to verify these models [8][10]. The above research indicates that the magnetic field attenuation of the LF EM wave in the plasma sheath is much smaller than the electric field attenuation. Therefore, we could utilize the magnetic field of the LF EM wave to maintain communications during radio blackout.

Ref. 11 conducted multiple shock tube experiments to simulate the plasma sheath, and then analyzed the steady-state effects of plasma on LF EM wave propagation, including steady attenuation and phase shift. In practice, the electron density of the plasma sheath is time-varying, resulting in a dynamic propagation process [12]-[14]. From the wireless communications perspective, the time-varying plasma is considered a propagation channel. The attenuation and phase shift characteristics caused by the channel are crucial prior knowledge, which helps build the channel model and determine communication schemes that can adapt to the channel characteristics. However, the dynamic effects on LF EM wave propagation have never been presented experimentally, and the attenuation and phase shift characteristics are still unclear.

The aim of this paper is to reveal the dynamic effects of timevarying plasma on LF EM wave propagation. In one shock tube experiment, we use the frequency division multiplexing (FDM) method to simultaneously propagate LF EM waves at four discrete frequencies $(1,3,10$, and $30 \mathrm{MHz})$ in a shared timevarying plasma. The time-varying attenuation, time-varying phase shift, and power spectrum at the four frequencies are observed to characterize the dynamic effects. These results reveal that the time-varying plasma interacts with LF EM waves in the time and frequency domains, which helps us deeply understand the propagation process of LF EM waves in timevarying plasma.
B. Sun, K. Xie, Y. Liu, Y. Zhang and S. Guo are with School of Aerospace Science and Technology, Xidian University, Xi'an 710071, China (e-mail: sunbin@stu.xidian.edu.cn; kaixie@mail.xidian.edu.cn; yanliu@xidian.edu.cn; yjzhang_53@stu.xidian.edu.cn; guoshaoshuail@163.com).

P. Ma is with the Hypervelocity Aerodynamics Institute, China Aerodynamics Research and Development Center, Mianyang 621000, China (e-mail: hbmaping@263.net). 


\section{EXPERIMENTAL PRINCIPLE}

Shock tubes are often used for generating non-steady plasma flows that can reach the desired reentry velocity. To investigate the dynamic effects of time-varying plasma on LF EM wave propagation, we use the FDM method to simultaneously propagate and measure LF EM waves at multiple frequencies in one shock tube experiment, which ensures identical timevarying behavior of the plasma. The experimental principle and photograph are shown in Fig. 1. In the experiment, the transmitted signal combines the four discrete frequency signals and is generated by an arbitrary function generator (AFG, AFG3102C, Tektronix). The time-domain waveform and power spectrum of the transmitted signal combining the four frequency signals are shown in Fig. 1(c). The LF EM waves at the four frequencies are emitted by a loop antenna, penetrate through the Teflon segment and time-varying plasma, and are then received by a magnetic field probe in the center of the shock tube. The output of the magnetic field probe is the received signal induced by closed coils arising from the magnetic field component of the LF EM waves. The received signal including the four frequencies is recorded by an oscilloscope (MSO54, Tektronix).

In the shock tube setup, the driver gas in the high-pressure chamber is ignited and breaks the aluminum diaphragm, forming a shock wave. Then, in the low-pressure chamber, the shock wave generates an unsteady plasma flow that reaches and envelopes the magnetic field probe. The time-varying plasma dynamically affects the propagation of the LF EM waves, causing time-varying attenuation, phase shift, and power spectrum distortion. During the time period affected by the time-varying plasma, the oscilloscope records the received signal, and the electrostatic probes measure the time-varying electron density.

The time-varying attenuation and phase shift of the LF EM waves at each frequency are different due to the dispersion property of plasma. Assuming that a frequency component of the LF EM wave is $f_{\mathrm{c}}$, for $f_{\mathrm{c}} \in[1,3,10,30]$ (units: MHz), the time-varying normalized amplitude $r(t)$ at $f_{\mathrm{c}}$ is defined as $r(t)=V(t) / V_{0}$, where $V_{0}$ is the initial amplitude of the received signal at $f_{\mathrm{c}}$ without the time-varying plasma influence (units: $\mathrm{V}$ ) and $V(t)$ is the time-varying amplitude of the received signal at $f_{\mathrm{c}}$ with the time-varying plasma influence (units: V). $A(t)$ represents the decibel scale of $r(t)$ and is given by (1). The term "attenuation" on the decibel scale is defined as the absolute value of $A(t)$ (i.e., $|A(t)|)$

$$
A(t)=20 \log _{10}(r(t))=20 \log _{10}\left(\frac{V(t)}{V_{0}}\right) .
$$

The time-varying phase shift is studied. Without the timevarying plasma influence, it is assumed that the phase of the received signal at $f_{\mathrm{c}}$ is $\theta_{0}$. The initial phase of a reference signal at $f_{\mathrm{c}}$ is the same as that of the received signal at $f_{\mathrm{c}}$. With the timevarying plasma influence, the phase of the received signal lags the phase of the reference signal, forming a phase shift. The time-varying phase shift $\varphi(t)$ at $f_{\mathrm{c}}$ is expressed as the difference between the phase of the received signal and the phase of the reference signal,

$$
\varphi(t)=\frac{360}{2 \pi} \cdot\left(\theta(t)-\theta_{0}\right),
$$

where $\theta(t)$ is the time-varying phase of the received signal at $f_{\mathrm{c}}$ (units: rad) and $\theta_{0}$ is the phase of the reference signal at $f_{\mathrm{c}}$ (units: $\mathrm{rad})$.

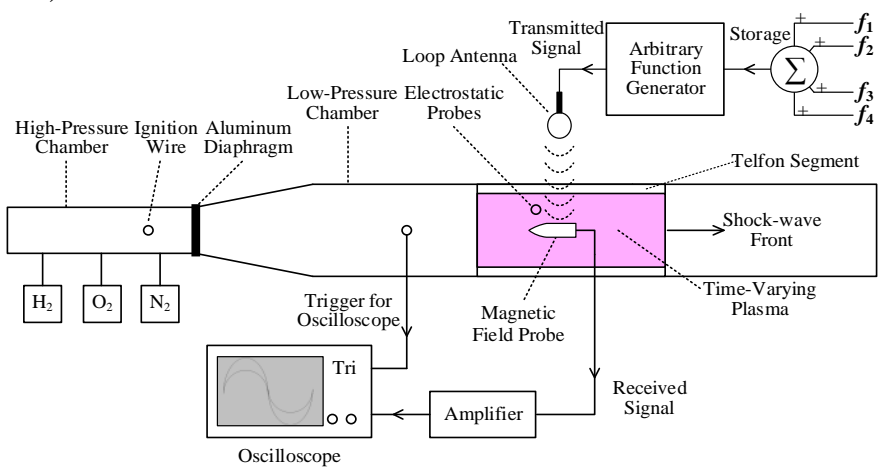

(a)

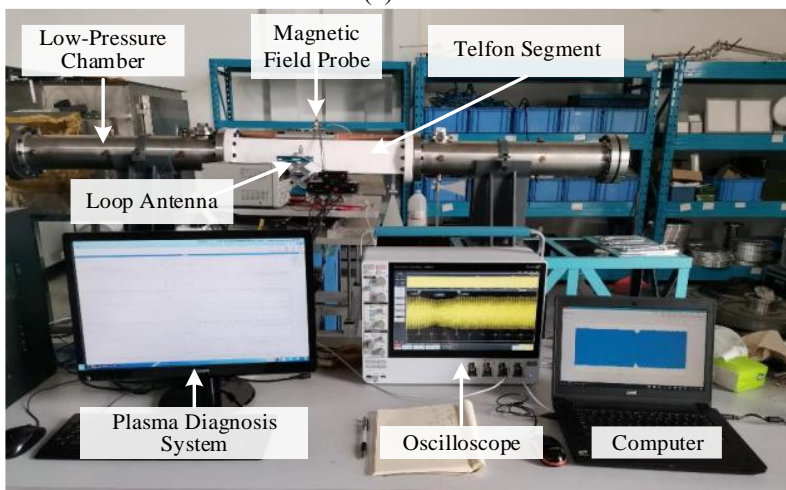

(b)

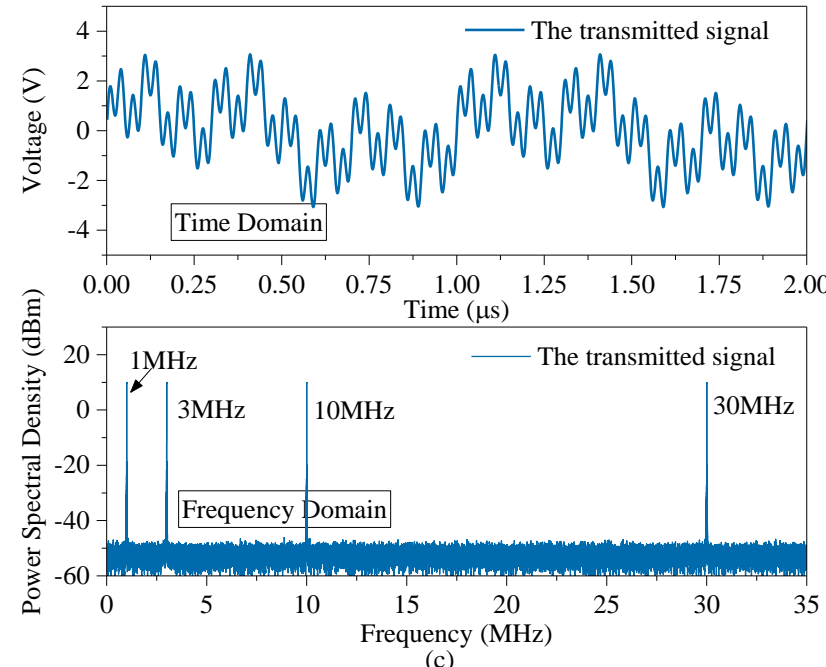

(c)

Fig. 1. (a) Principle and (b) photograph of the shock tube experiment; (c) timedomain waveform and power spectrum of the transmitted signal.

\section{EXPERIMENTAL RESULTS AND DISCUSSION}

In the shock tube experiment, the plasma parameter results are that the electron density mainly fluctuates in the range of $1.20 \times 10^{13}$ to $2.0 \times 10^{13} / \mathrm{cm}^{3}$, the collision frequency is approximately $9.8 \mathrm{GHz}$, the electronic temperature is $5800 \mathrm{~K}$, the velocity of the shock wave is $6 \mathrm{~km} / \mathrm{s}$, and the plasma duration is approximately $40 \mu \mathrm{s}$. 
Figure 2 shows the raw data $S(t)$ of the received signal, timevarying electron density, normalized amplitude $A(t)$ and phase shift $\varphi(t)$ in the time domain. Since the electron density is timevarying, the attenuation and phase shift of the LF EM waves are also time-varying. At each moment, the attenuation and phase shift at the four frequencies correspond to the same electron density. The experimental results indicate that the time-varying attenuation and phase shift are dependent on the time-varying behavior of the electron density. As the electron density increases, the attenuation and phase shift of the LF EM waves increase. During the entire experiment, the attenuation at 1 $\mathrm{MHz}$ and $3 \mathrm{MHz}$ does not exceed $0.5 \mathrm{~dB}$, and the phase shift does not exceed $25^{\circ}$, where the electron density is $1.97 \times 10^{13}$ $/ \mathrm{cm}^{3}$ and the collision frequency is $9.8 \mathrm{GHz}$. These results reflect that the LF EM waves at $1 \mathrm{MHz}$ and $3 \mathrm{MHz}$ penetrate the time-varying high-density plasma with small attenuation and phase shift.

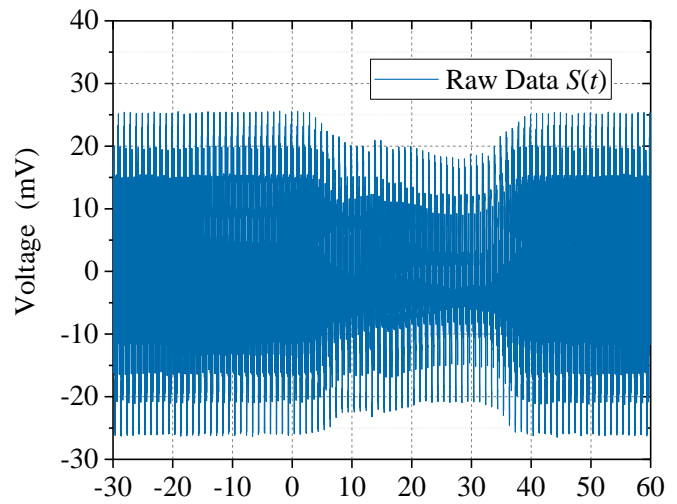

(a) Time $(\mu \mathrm{s})$

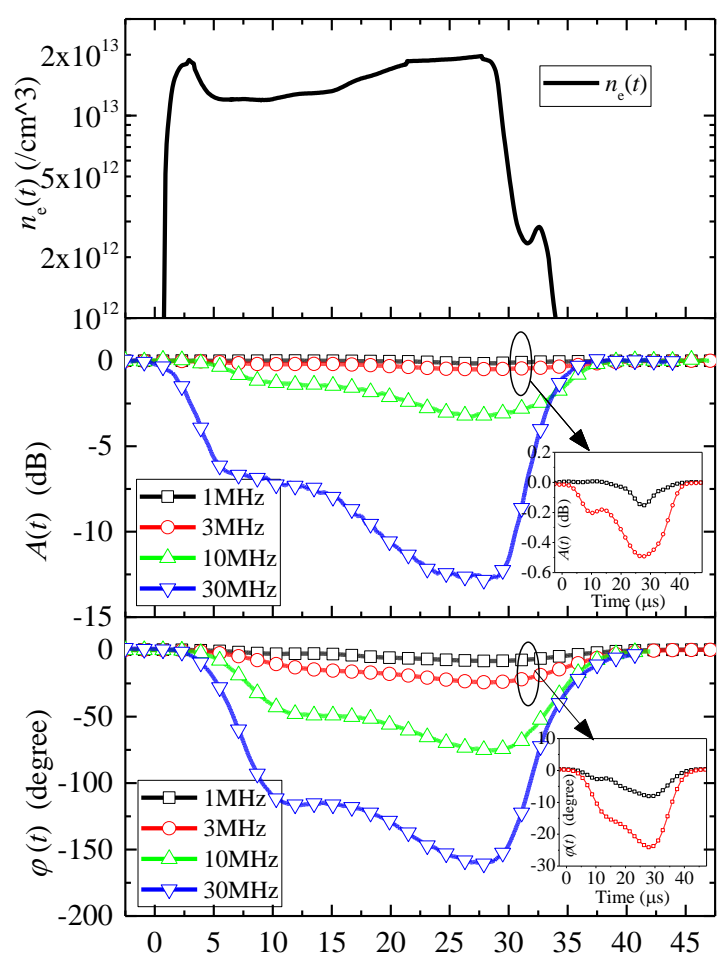

(b) Time ( $\mu \mathrm{s})$

Fig. 2. (a) Raw data $S(t)$ of the received signal including the four frequencies; (b)experimental results of the electron density, normalized amplitude $A(t)$ and phase shift $\varphi(t)$.
Furthermore, because the plasma is a dispersive medium, as the frequency of the LF EM waves increases, their maximum attenuation and phase shift increase. The maximum attenuation at $30 \mathrm{MHz}$ does not exceed $13 \mathrm{~dB}$, and the maximum phase shift does not exceed $-170^{\circ}$. When the fluctuation range of the electron density at the four frequencies is identical, as the frequency of LF EM waves increases, the fluctuation ranges of the attenuation and phase shift increases, as shown in Fig. 2. These time-varying attenuation and phase shift characteristics are crucially important to establish the plasma channel model and to identify communication schemes for alleviating the dynamics of the plasma channel.

With reference to the numerical simulation model of a shock tube experiment [11], the theoretical results of the normalized amplitude and phase shift are shown in Fig. 3. The experimental results corresponding to three electron densities are also plotted in Fig. 3, where the three electron densities are 1.3, 1.6, and $1.9 \times 10^{13} / \mathrm{cm}^{3}$. Figure 3 indicates that the experimental results are well consistent with the theoretical results, which demonstrates the validity of the experiment.
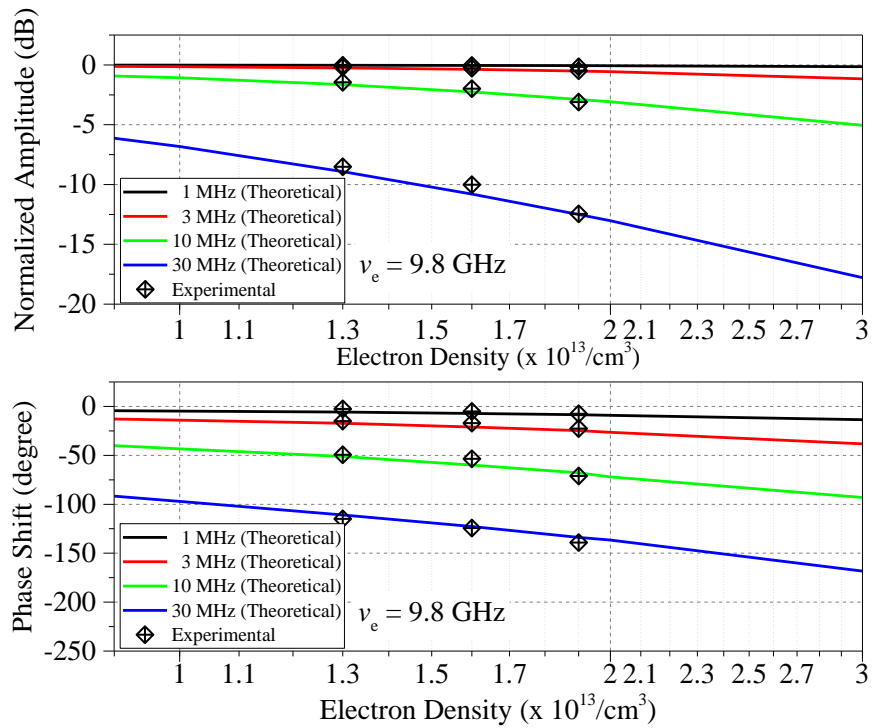

Fig. 3. Theoretical results of the normalized amplitude and the phase shift in the time-varying plasma, and corresponding experimental results. The experimental results are obtained from Fig. 2, where the three electron densities are $1.3,1.6$, and $1.9 \times 10^{13} / \mathrm{cm}^{3}$ and the three time moments are $14.2,18.8$, and $25.3 \mu \mathrm{s}$.

In addition to the above effects in the time domain, the dynamic effects in the frequency domain are also investigated. The time series of the received signal within $0 \sim 40 \mu$ s were analyzed by using the periodogram method to obtain its power spectrum affected by the time-varying plasma [15]. Figure 4 shows the power spectral density (PSD) at the four frequencies with and without the time-varying plasma influence. The PSD amplitudes at the four frequencies with the time-varying plasma influence are lower than those without the time-varying plasma influence. The plasma causes a power reduction at the four frequencies. As the frequency of the LF EM waves increases, the attenuation of the PSD amplitude increases (i.e. $A_{\text {PSD }}$ in Fig. 4), which is consistent with the attenuation characteristics in the time domain.

Moreover, the PSDs without the time-varying plasma 
influence are narrow peak, but the PSDs at the four frequencies with the time-varying plasma influence are all expanded. The spectrum expansion is positively related to the frequency of the LF EM waves. As the frequency of the LF EM waves increases, the spectrum expansion becomes wider. When the PSD is equal to $-80 \mathrm{dBm}$, the bandwidths of the spectrum expansion are $120.8,179.2,245.8$, and $280.2 \mathrm{kHz}$ at $1,3,10$, and $30 \mathrm{MHz}$, respectively.

The physical mechanism of the spectrum expansion phenomenon is that the propagation process of LF EM waves dynamically couples the characteristics of the turbulence flow

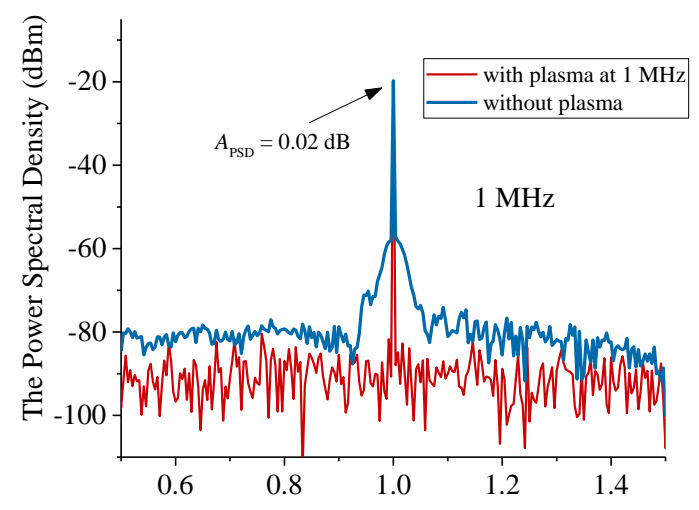

(a) Frequency $(\mathrm{MHz})$

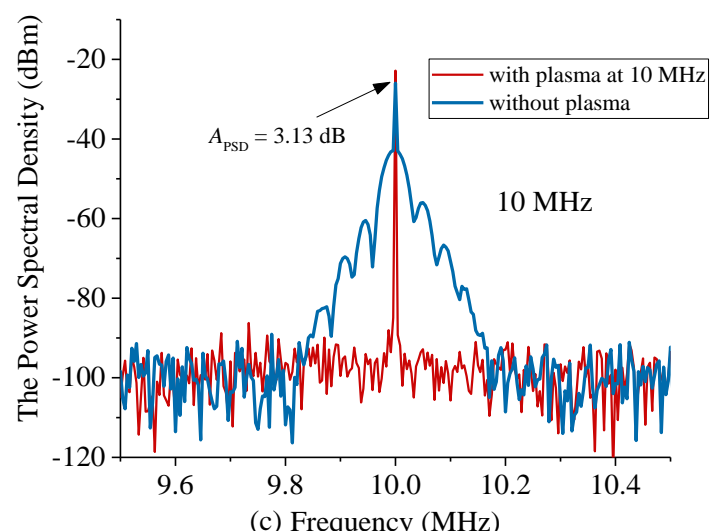

field in the time-varying plasma [16]-[17]. The time-varying plasma acts as a time-varying propagation channel, which leads to the spectrum expansion phenomenon. In the experiment, the time-varying behavior of the plasma at the four frequencies is identical due to the use of the FDM method. As the frequency of the LF EM wave increases, the attenuation and phase shift at the frequency increase, so the spectrum expansion becomes wider. Therefore, the time-varying behavior of the plasma dynamically affects the propagation of the LF EM waves, which causes time-varying attenuation, time-varying phase shift and spectrum expansion of the LF EM waves.

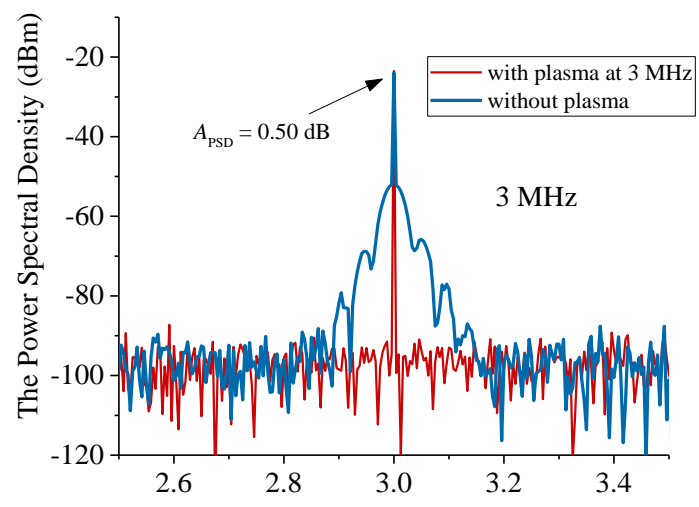

(b) Frequency $(\mathrm{MHz})$

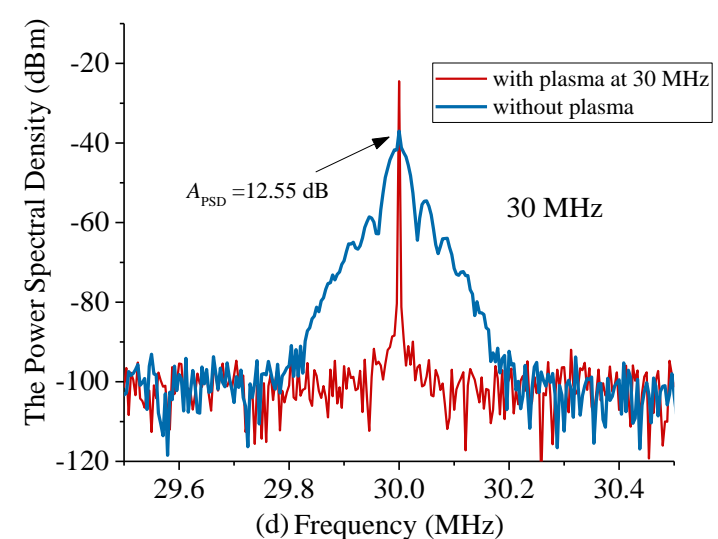

Fig. 4. Power spectrum of the received signal at (a) $1 \mathrm{MHz}$, (b) $3 \mathrm{MHz}$, (c) $10 \mathrm{MHz}$, and (d) $30 \mathrm{MHz}$ with and without the time-varying plasma influence.

\section{CONCLUSION}

In this paper, we reveal the dynamic effects of timevarying plasma on the propagation of LF EM waves through a shock tube experiment. In the experiment, the FDM method is applied so that LF EM waves at four discrete frequencies simultaneously penetrate through a shared time-varying plasma, and then, their time-varying attenuation, time-varying phase shift, and power spectrum are presented. The timevarying attenuation and phase shift are strongly dependent on the time-varying behavior of the plasma. As the electron density increases (decreases), the attenuation and phase shift of the LF EM increase (decreases). The fluctuation ranges of the attenuation and phase shift increases with the frequency of LF EM waves. Furthermore, in the frequency domain, the power spectrum of the LF EM waves is expanded due to the dynamic effects of the time-varying plasma. The bandwidth of spectrum expansion is positively related to the frequency of the LF EM waves. These experimental results are crucially important for designing LF communication systems. Finally, our future study will focus on establishing the plasma channel model and on evaluating the LF communication performance under various digital modulation schemes.

\section{REFERENCES}

[1] J. P. Rybak and R.J. Churchill, "Progress in Reentry Communications," IEEE Transactions on Aerospace and Electronic Systems, vol. AES-7, no. 5, pp. 879-894, Sept. 1971.

[2] Y. Sha, H. Zhang, X. Guo and M. Xia, "Analyses of Electromagnetic Properties of a Hypersonic Object With Plasma Sheath," IEEE Transactions on Antennas and Propagation, vol. 67, no. 4, pp. 2470-2481, Apr. 2019. 
[3] A. V. Bogatskaya, E. A. Volkova, N. V. Klenov, M. V. Tereshonok and A. M. Popov, "Toward the Nonstationary Theory of a Telecommunication Channel Through a Plasma Sheath," IEEE Transactions on Antennas and Propagation, vol. 68, no. 6, pp. 4831-4838, June 2020.

[4] J.F. Liu, H. Lv, Y.C. Zhao, Y. Fang and X.L. Xi, "Stochastic PLRC-FDTD Method for Modeling Wave Propagation in Unmagnetized Plasma," IEEE Antennas and Wireless Propagation Letters, vol. 17, no. 6, pp. 1024-1028, Jun. 2018.

[5] E. D. Gillman, J. E. Foster and I. M. Blankson, "Review of Leading Approaches for Mitigating Hypersonic Vehicle Communications Blackout and a Method of Ceramic Particulate Injection Via Cathode Spot Arcs for Blackout Mitigation," NASA Technical Report, NASA/TM-2010-216220, E-17194, NASA, Washington, DC, USA, 2010.

[6] Minkwan Kim, Iain D. Boyd, and Michael Keidar, "Modeling of Electromagnetic Manipulation of Plasmas for Communication During Reentry Flight," Journal of Spacecraft and Rockets, vol. 47, no. 1, pp. 2935, Jan., 2010.

[7] X. Lyu, C. Jiang, W. Feng and N. Ge, "A Shock Tube Experimental System for Communication Performance Evaluation Under the Time-Varying Plasma Flow Channel," IEEE Transactions on Plasma Science, vol. 45, no. 9, pp. 2450-2459, Sept. 2017.

[8] D.L. Liu, X.P. Li, K. Xie, and Z.W. Liu, "The propagation characteristics of electromagnetic waves through plasma in the near-field region of lowfrequency loop antenna," Physics of Plasmas, vol. 22, no. 10, pp. 102106, Oct. 2015.

[9] D.L. Liu, X.P. Li, Y.M. Liu, K. Xie, and B.W. Bai, "Attenuation of lowfrequency electromagnetic wave in the thin sheath enveloping a high-speed vehicle upon re-entry," Journal of Applied Physics, vol. 121, no. 7, pp. 074903, Feb. 2017.
[10] K. Xie, S.S. Guo, B. Sun, L. Quan, and Y. Liu, "Modeling and experimental study of low-frequency electromagnetic wave propagation in cylindrical enveloping plasma produced by a shock tube," Physics of Plasmas, vol. 26, no. 7, pp. 073509, Jul. 2019.

[11] K. Xie, B. Sun, S.S. Guo, L. Quan, and Y. Liu, "Experimental apparatus for investigating the propagation characteristics of the low-frequency electromagnetic waves in hypersonic plasma fluid generated by shock tube," Review of Scientific Instruments, vol. 90, no.7, pp. 073503, Jul. 2019.

[12] B. Yao, X.P. Li, L. Shi, Y.M. Liu, and B.W. Bai, "A layered fluctuation model of electron density in plasma sheath and instability effect on electromagnetic wave at Ka band," Aerospace Science and Technology, vol.78, pp. 480-487, Jul. 2018.

[13] G. He, Y. Zhan, N. Ge, Y. Pei and B. Wu, "Measuring the Time-Varying Channel Characteristics of the Plasma Sheath From the Reflected Signal," IEEE Transactions on Plasma Science, vol. 42, no. 12, pp. 3975-3981, Dec. 2014.

[14] B. Sun, K. Xie, L. Shi, M. Yang, B. Yao and S. Guo, "Experimental Investigation on Electromagnetic Waves Transmitting Through Exhaust Plume: From Propagation to Channel Characteristics," IEEE Transactions on Antennas and Propagation, Early Access.

[15] Stoica, Petre, and Randolph L. Moses, Spectral Analysis of Signals, Prentice Hall, Englewood Cliffs, NJ, USA, 2005.

[16] S. P. Sharma and C. Park, "Operating characteristics of a $60-$ and $10-\mathrm{cm}$ electric arc-driven shock tube-Part I: The driver," Journal of Thermophysics and Heat Transfer, vol. 4, no. 3, pp. 259-265, Jul. 1990.

[17] O. Igra and F. Seiler, Experimental Methods of Shock Wave Research, Springer, Boston, MA, USA, 2016. 